\title{
Thin film PZT-Si structure with quadrant-diagonal electrode system as an element of position sensitive pyroelectric detector
}

\author{
S.L. Bravina'), E. Cattan $^{2)}$, N.V. Morozovsky ${ }^{1)}$, D. Remiens ${ }^{2)}$ \\ 1) Institute of Physics, NAS of Ukraine, 46 prospect Nauki, 03028 Kyiv, Ukraine \\ e-mail: bravina@iop.kiev.ua \\ 2) LAMAC-dept. MIMM, Universite de Valenciennes et du Hainaut-Cambresis, CRITT-Z.I. Champ de l'Abbesse, 59600 Maubeuge, France \\ e-mail: eric.cattan@univ.valenciennes.fr
}

\begin{abstract}
The main characteristics of position sensitive systems of pyroelectric detectors of radiation (PDR) with sensitive elements based on PZT film on Si-substrate were investigated by photopyromodulation method. Pt-PZT-Pt/Ti-SiO $2 / \mathrm{Si}$ structures with $1.9 \mu \mathrm{m}$ (111)-oriented PZT (54/46) layer were manufactured by R.F. magnetron sputtering. Top Pt electrodes in a shape of isosceles rectangular triangle together make the quadrant-diagonal system. The results of pyroelectric undersurface probing and "along surface" pyroelectric scanning show that the investigated quadrant-diagonal system of SE makes it possible to examine all main types of differential position-sensitive PDR. The experimental results for investigated 2element single-coordinate PDR variants are in a good agreement with calculations for the corresponding systems.
\end{abstract}

Keywords: PZT-ceramic films, quadrant-diagonal electrode system, position-sensitivity, pyroelectric detectors, photopyromodulation method.

Paper received 10.12.01; revised manuscript received 08.02.02; accepted for publication 05.03.02.

\section{Introduction}

Since the first application of ceramics of PZT type for pyroelectric detectors of radiation (PDR) [1] the transition from volume to thin layer configurations of sensitive elements (SE) of PDR came over [2]. At the same time took place the transition from single element PDR and their 2- and 4- element and matrix assembling to $1 \times 2$ and $2 \times 2$ chips and multi-element systems based on thin films of PZT on Si-substrate. Progress in this direction was stimulated by needs of thermal field monitoring and design of IR-spectroscopic systems and systems of IR-orientation. For systems of controlling the spatial position of IR-laser beams and systems of IR-orientation were developed position sensitive PDR [3] whose output signal depends on the position (coordinate) of the energetic centre of radiation beam cross-section relative to the zero point of the PDR.

For more than 40 year term of using ceramics of PZT type proved itself as one of the best for creating stable polar-active materials resistant to thermal impacts and repolarized in a relatively easy way [4]. At present PZTceramics are considered as one of the most suitable material for SE of position sensitive PDR. It is connected both with variety of new methods of manufacturing effective pyroactive PZT films on Si-substrate and ease of matching miniature SE of PDR with silicone field effect transistor (Si-FET).

In current literature on characteristics of PDR maximal attention is given to single- and multi- element PDR of usual type and the information about position-sensitive PDR is extremely limited. 
In this paper we present the results of investigation of the main characteristics of position-sensitive systems of PDR with SE based on PZT film on Si-substrate.

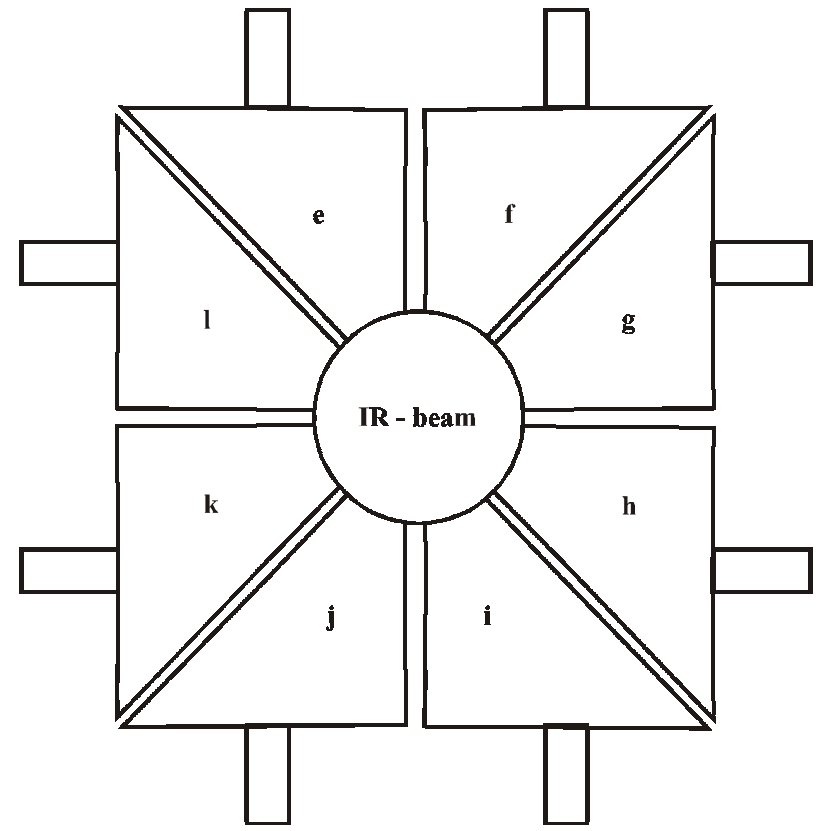

a)

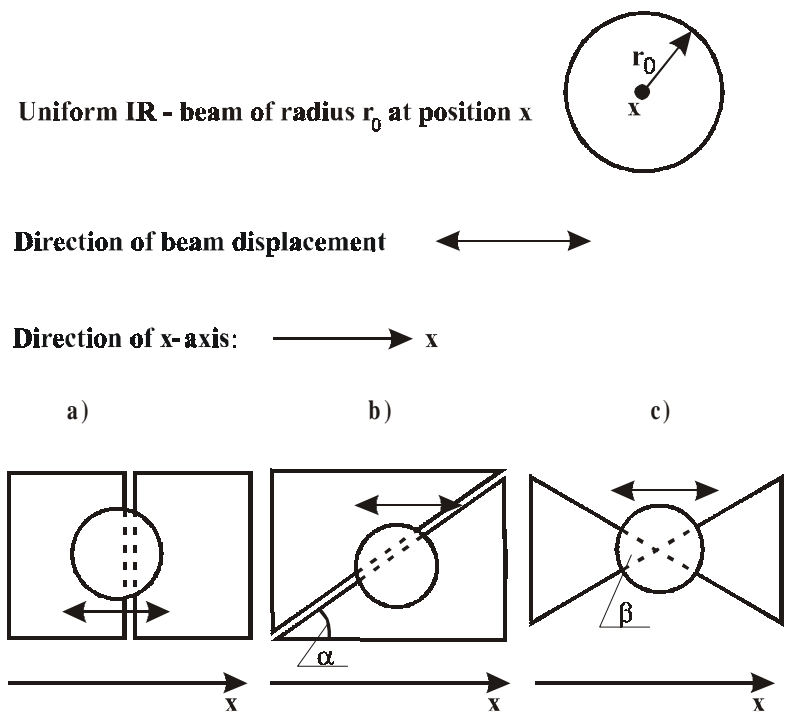

Fig. 1. Schematic view of:

(a) - quadrant-diagonal electrode system of position sensitive pyroelectric element;

(b) - side-to-side configuration of SE electrode system;

(c) - diagonal configuration of SE electrode system;

(d) - top-to-top triangle configuration of SE electrode system

\section{Experimental}

Pt-PZT-Pt/Ti-SiO $2 / \mathrm{Si}$ structures with $1.9 \mu \mathrm{m}$ (111)oriented PZT (54/46) layer were manufactured by R.F. magnetron sputtering. Top Pt- electrodes (deposited by sputtering followed by a lift-off) in a shape of isosceles rectangular triangles together make the quadrant-diagonal system (Fig. 1a). The area of each triangle is $\approx 1 \mathrm{~mm}^{2}$; the common dimensions of electrode system are near $4 \mathrm{x} 4$ $\mathrm{mm}$. Each irradiated element has current-carrying thin stripe with circle current electrode of $\approx 1 \mathrm{~mm}$ diameter.

Experiments were performed on the different pairs of SE of quadrant-diagonal system of PDR (see Fig. 1a). The investigated configuration of SE makes it possible to examine all main types of differential position-sensitive PDR. By means of paired combinations can be realized 2-element single-coordinate PDR variants and by means of two-paired combinations are realized quadrant 4-element two-coordinate position sensitive PDR variants, for example "h-g", "h-k", "f-k" combinations.

The measurements of electrical capacitance $\mathrm{C}_{\mathrm{s}}$ and electrical conductance $\mathrm{G}_{\mathrm{s}}$ of all the triangle elements of investigated system at $1 \mathrm{kHz}$ showed that $\mathrm{C}_{\mathrm{s}} \approx 5 \mathrm{nF}, \mathrm{G}_{\mathrm{s}} \approx$ $0,5 \mu \mathrm{S}$ with the deviation $\leq 10 \%$ for each of the elements "e-...-k".

Measurements of the pyroelectric response were performed with the measuring set described in Ref. 5 by means of pyroelectric photomodulation method.

For measurements of amplitude-to-frequency $U_{\pi}(f)$ and phase-to-frequency $\varphi_{\pi}(\mathrm{f})$ characteristics of the pyroelectric response the sample under investigation was connected to the matching stage with step-varying input impedance from high value $\sim 10 \mathrm{G} \Omega$ at $20 \mathrm{~Hz}$ to low one $\sim 100 \mathrm{k} \Omega[6]$.

During the measurements the sample was irradiated by modulated IR-probe from IR LED (light emitting diode) supplied by generator of sinusoidal voltage through the matching stage.

\section{Basic relations}

\subsection{Single Element PDR}

In the case of a free sample of pyroelectric material in the shape of plate with metal electrodes on its main polar surfaces $[2,3,5]$

$$
\mathrm{U}_{\mathrm{p}}=(\gamma / \mathrm{c}) \alpha \Phi_{0} \mathrm{~A}_{0} \mathrm{R}_{\mathrm{e}} / \mathrm{d} \sqrt{1+\left(\omega_{\mathrm{m}} \mathrm{R}_{\mathrm{e}} \mathrm{C}_{\mathrm{e}}\right)^{2}}
$$

where $\Phi_{0}$ is the incident sinusoidal modulated thermal flux, $\omega_{\mathrm{m}}=2 \pi \mathrm{f}_{\mathrm{m}}, \mathrm{f}_{\mathrm{m}}$ is the modulation frequency, $\mathrm{A}_{0}$ is the area of the irradiated surface, $a$ is the absorptivity, $\mathrm{C}_{\mathrm{e}}=\mathrm{C}_{\mathrm{L}}+\mathrm{C}_{\mathrm{s}}, \mathrm{R}_{\mathrm{e}}^{-1}=\mathrm{R}_{\mathrm{L}}^{-1}+\mathrm{R}_{\mathrm{s}}^{-1}$, where $\mathrm{R}_{\mathrm{L}}$ and $\mathrm{C}_{\mathrm{L}}$ are the electrical resistance and capacitance in the external 


\section{S.L. Bravina et al.: Thin film PZT-Si structure with quadrant-diagonal electrode system...}

circuit of the $\mathrm{SE}, \mathrm{R}_{\mathrm{s}}=\mathrm{d} / \sigma \mathrm{A}_{\mathrm{e}}$ and $\mathrm{C}_{\mathrm{s}}=\varepsilon \varepsilon_{0} \mathrm{~A}_{\mathrm{e}} / \mathrm{d}$ are the electrical resistance and capacitance of the $\mathrm{SE}, \mathrm{A}_{\mathrm{e}}$ is the electrode area, $d$ is the thickness of the SE, $\gamma, c, \varepsilon$ and $\sigma$ are the pyroelectric coefficient, volume heat capacity, dielectric permittivity and conductivity of SE material respectively, and $\varepsilon_{0}=8,85 \cdot 10^{-12} \mathrm{~F} / \mathrm{m}$.

It results from (1) that in the pyroelectric current mode when $\omega_{\mathrm{m}} \mathrm{R}_{\mathrm{e}} \mathrm{C}_{\mathrm{e}}<<1$

$$
\mathrm{U}_{\pi}=\mathrm{U}_{\pi 1}=(\gamma / \mathrm{c}) \alpha \Phi_{0} \mathrm{~A}_{0} \mathrm{R}_{\mathrm{L}} / \mathrm{d}
$$

and $\mathrm{U}_{\pi 1} \propto(\gamma / \mathrm{c}) / \mathrm{d}, \mathrm{U}_{\pi 1}=\operatorname{const}\left(\mathrm{f}_{\mathrm{m}}\right)$. At that $\varphi_{\pi}=\varphi_{\pi 1}=$ const $\left(\mathrm{f}_{\mathrm{m}}\right)$ and $\mathrm{U}_{\pi 1}$ is in phase with thermal flux intensity.

In the pyroelectric voltage mode when $\omega_{\mathrm{m}} \mathrm{R}_{\mathrm{e}} \mathrm{C}_{\mathrm{e}}>>1$

$\mathrm{U}_{\pi}=\mathrm{U}_{\pi 2}=(\gamma / \mathrm{c} \varepsilon) \alpha \Phi_{0} \mathrm{~A}_{0} / \omega_{\mathrm{m}} \varepsilon_{0} \mathrm{~A}_{\mathrm{e}}$

and $\mathrm{U}_{\pi 2} \propto(\gamma / \mathrm{c} \varepsilon) / \mathrm{f}_{\mathrm{m}}, \mathrm{U}_{\pi 2}=\operatorname{const}(\mathrm{d})$. At that $\varphi_{\pi}=\varphi_{\pi 2}=$ $\operatorname{const}\left(\mathrm{f}_{\mathrm{m}}\right)$ and $\mathrm{U}_{\pi 2}$ and thermal flux intensity have the phase shift equals $\pi / 2$, and so $\varphi_{\pi 2}-\varphi_{\pi 1}=\pi / 2$.

As follows from (1) - (3) the mode of $U_{\pi 1}$ measurement turns to $\mathrm{U}_{\pi 2}$ mode with increasing $\mathrm{f}_{\mathrm{m}}$ value and the transition frequency $f_{m t}$ is determined by relation $\omega_{m t} R_{e} C_{e}=1$. At frequencies above $\mathrm{f}_{\mathrm{mt}}$ only $\mathrm{U}_{\pi 2}$ mode is realized.

In the case of arrangement of the SE plate of the thickness $d$ on the heat removing substrate of thickness $L_{S}>>d$ under the condition that the length of the temperature wave $\lambda_{\mathrm{T}} \leq \mathrm{L}_{\mathrm{S}}\left(\lambda_{\mathrm{T}}=\left(a / \pi \mathrm{f}_{\mathrm{m}}\right)^{1 / 2}, a\right.$ is the thermal diffusivity)

$$
\begin{aligned}
& \mathrm{U}_{\pi 1}\left(\mathrm{f}_{\mathrm{m}}\right) \propto \mathrm{f}_{\mathrm{m}}^{1 / 2}, \\
& \mathrm{U}_{\pi 2}\left(\mathrm{f}_{\mathrm{m}}\right) \propto \mathrm{f}_{\mathrm{m}}^{-1 / 2},
\end{aligned}
$$

and for $\varphi_{\pi 1,2}$ values due to the heat removing action of substrate appears the addition value equal $-(\pi / 4)$.

The dependences of $U_{\pi 1,2}\left(f_{m}\right)$ are well described by (4a) and (4b) when the value of electrode area $A_{e}$ is near the same as the substrate surface area $A_{S}$ and for $A_{S}>>A_{e}$ the effect of lateral heat spreading into substrate should be taken into consideration. At that the frequency dependence of the radius of heat spread area is of the same type as that for the heat penetration depth and these factors both determine the dependences of $U_{\pi 1,2}\left(f_{m}\right)$.

At $\lambda_{\mathrm{T}} \leq d$ the influence of the substrate becomes insignificant and when $\lambda_{\mathrm{T}}<<d$ instead of (4a) and (4b) the dependences (2) and (3) for $\mathrm{U}_{\pi 1,2}\left(\mathrm{f}_{\mathrm{m}}\right)$ of a free pyroactive plate are realized.

Thus, any thermal non-uniformity placed at the depth close to $\lambda_{T}$ value is manifested as a peculiarity of $U_{\pi 1,2}\left(f_{m}\right)$ and $\varphi_{\pi 1,2}\left(f_{m}\right)$. So, in general case, pyroelectric undersurface probing makes it possible to conclude by analyzing shapes of $\mathrm{U}_{\pi 1,2}\left(\mathrm{f}_{\mathrm{m}}\right)$ and $\varphi_{\pi 1,2}\left(\mathrm{f}_{\mathrm{m}}\right)$ whether there is the thermal non-uniformity and what the character of pyroactivity distribution is (e.g. near-surface or volumetric).

\subsection{Double Element Position Sensitive PDR}

In the case of the arrangement of two SE on the substrate with one common electrode under series or parallel connection of these SE the position sensitive PDR system of differential type is formed. Under simultaneous irradiation of both SE their signals of pyroelectric response are opposite in phase. That is why under the probe through the boundary which divides the SE electrodes the changes of $U_{\pi 1,2}$ and $\varphi_{\pi 1,2}$ corresponding to the change of portion of thermal flux impacted on each SE are observed.

So, "along surface" pyroelectric scanning makes it possible to determine the position characteristics of the system of SE irradiated by one beam-probe.

The position characteristics of $U_{\pi}$ can be determined by consideration of changes of effective illuminated area of SE of position sensitive PDR under displacement the beam-probe from one to another SE.

For the simplest 2-element differential system in the case of uniform distribution of energy under cross section of beam-probe of round section of radius $r_{0}$ and coordinate of the centre of beam cross-section $x$ measured relative to zero-signal point of the system were obtained the following dependences $\mathrm{U}_{\pi}(x)$ :

1. For a side-to-side configuration of SE system when the direction of displacement of probe beam is perpendicular to the boundary of electrode division (see Fig. 1b)

$$
\mathrm{U}_{\pi}(x) \propto \mathrm{U}_{\pi}\left[\left(x / \mathrm{r}_{0}\right) \sqrt{1-\left(x / \mathrm{r}_{0}\right)^{2}}+\arcsin \left(x / \mathrm{r}_{0}\right)\right]
$$

2 . For a diagonal configuration of SE system when the direction of displacement of probe beam makes the angle $\alpha<90^{\circ}$ with the boundary of electrode division (see Fig. 1c)

$\mathrm{U}_{\pi}(x) \propto \mathrm{U}_{\pi}\left[\left(x \sin \alpha / \mathrm{r}_{0}\right) \sqrt{1-\left(x \sin \alpha / \mathrm{r}_{0}\right)^{2}}+\arcsin \left(x \sin \alpha / \mathrm{r}_{0}\right)\right]$

3. For a top-to-top triangular configuration of SE system when the probe-beam is displacing along the direction of heights of triangle electrodes of SE with angle $\mathrm{b}$ at the vertex of the triangles (see Fig. 1d)

$\mathrm{U}_{\pi}(x) \propto \mathrm{U}_{\pi}\left[\left(x \sin (\beta / 2) / \mathrm{r}_{0}\right) \sqrt{\left.1-\left(x \sin (\beta / 2) / \mathrm{r}_{0}\right)^{2}+\arcsin \left(x \sin (\beta / 2) / \mathrm{r}_{0}\right)\right]}\right.$

\section{Results and Discussion}

\subsection{Single Element PDR}

The investigated dependences of $U_{\pi 1,2}\left(f_{m}\right)$ and $\varphi_{\pi 1,2}\left(\mathrm{f}_{\mathrm{m}}\right)$ are found to be identical for any "e-k" elements and are presented in Fig. 2a and Fig. 2b. Small scatter of $\mathrm{U}_{\pi 1,2}$ values can be connected with the difference in $\mathrm{C}_{\mathrm{s}}$ and $R_{S}$ values in consequence of the difference in the degree of unipolarity of the elements and scatter of absorptivity of the electrodes. Repolarization of SE gives $180^{\circ}-$ addition to $\varphi_{\pi 1,2}$ which corresponds to the change of sign of pyroelectric reaction of SE and only insignificant variations of $U_{\pi 1,2}\left(f_{m}\right)$ and $\varphi_{\pi 1,2}\left(f_{m}\right)$ dependences (compare Fig. 2a and Fig. 2b).

The "substrate effect" is well visible due to the presence of negative addition to $\varphi_{\pi 1,2}$ which is about $-45^{\circ}$, diffuse maximum of $U_{\pi 1}\left(f_{m}\right)$ and fold of $U_{\pi 2}\left(f_{m}\right)$ (see Fig. 2a, b). 

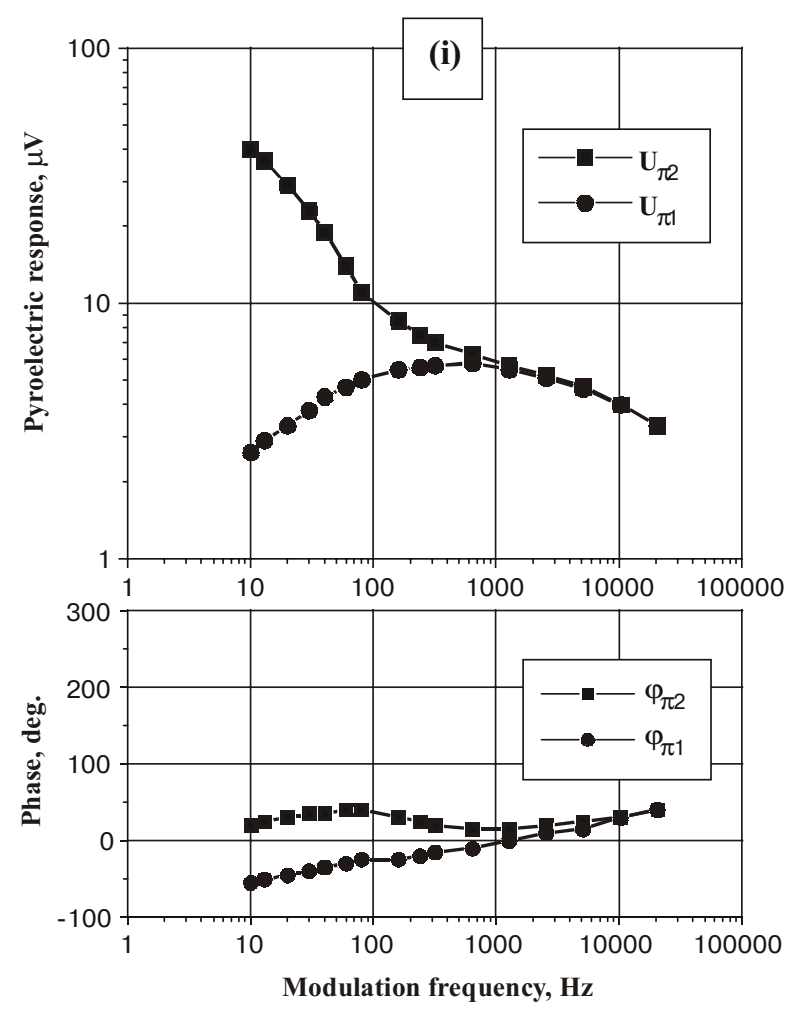
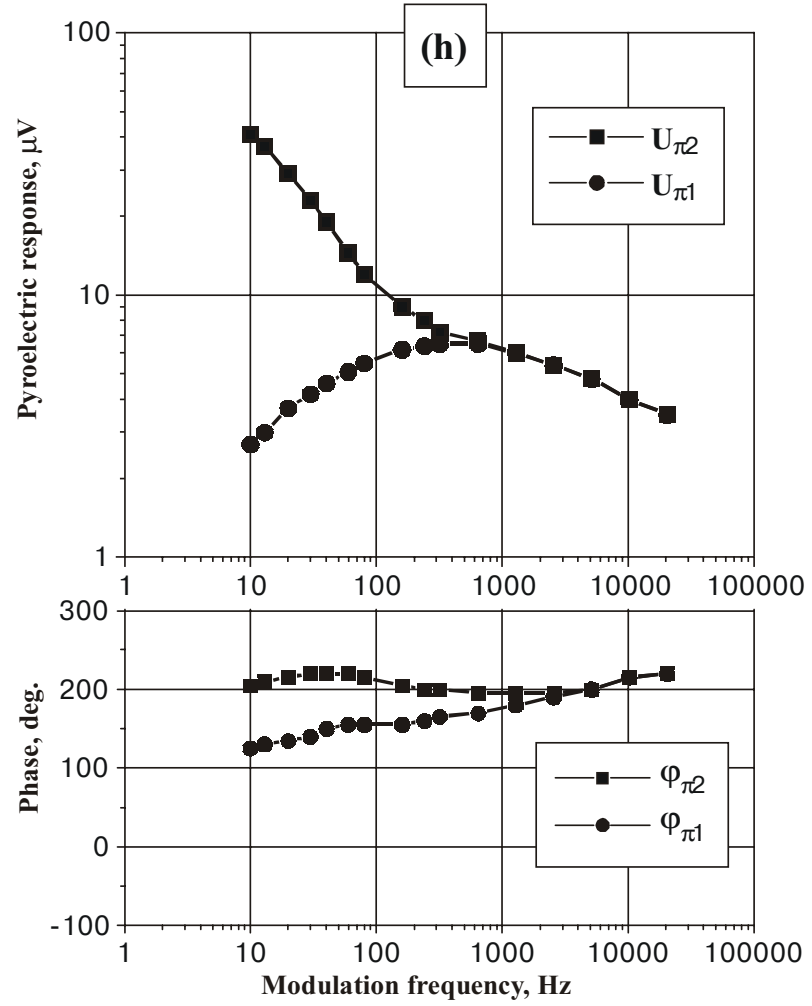

Fig. 2. The dependences of $\mathrm{U}_{\pi 1,2}(\mathrm{f})$, and $\varphi_{\pi 1,2}(\mathrm{f})$ of $1.9 \mathrm{~mm}$ PZT- film with top Pt-electrode and bottom Pt/Ti-electrode on the $350 \mathrm{~mm}$ $\mathrm{SiO}_{2} / \mathrm{Si}$-substrate:

a - for i-element after $+35 \mathrm{~V}, 5$ min DC poling;

b - for h-element after - $35 \mathrm{~V}, 5 \mathrm{~min}$ DC poling.

The critical frequency regions connected with the "substrate effect" should be evaluated by the comparison of $\lambda_{\mathrm{T}}$, effective electrode size $\mathrm{r}_{\mathrm{e}}$ and $\mathrm{L}_{\mathrm{S}}$ values.

Indeed, one of the critical frequency region when lateral heat spreading directed along the substrate surface decreases is near the frequency $\mathrm{f}_{\mathrm{mc}}{ }^{*}$ which value corresponds to the condition $\lambda_{\mathrm{T}}=\mathrm{r}_{\mathrm{e}}$. The estimation of $\mathrm{f}_{\mathrm{mc}}{ }^{*}$ with known electrode area $\left(\mathrm{r}_{\mathrm{e}}=\sqrt{\mathrm{A}_{\mathrm{e}}} / \pi \approx 0.5 \mathrm{~mm}\right)$ and thermal diffusivity for crystalline $\mathrm{Si}\left(\mathrm{a}_{\mathrm{T}} \approx 0.9 \cdot 10^{-4} \mathrm{~m}^{2} / \mathrm{s}\right)$ gives $\mathrm{f}_{\mathrm{mc}}{ }^{*} \approx 120 \mathrm{~Hz}$

Another critical frequency region is connected with the transition from "through substrate mode" ( at which the heated volume of substrate decreases with increasing $f_{m}$ value only due to decrease of lateral spreading) to "inside substrate mode" (at which the heated volume of substrate decreases with increasing $\mathrm{f}_{\mathrm{m}}$ value both due to decrease of lateral spreading distance and heat penetration depth along the substrate thickness). It lies near the frequency $\mathrm{f}_{\mathrm{mc}}{ }^{* *}$ which corresponds to the condition $\lambda_{\mathrm{T}}=$ $\mathrm{L}_{\mathrm{S}}$. The estimation of $\mathrm{f}_{\mathrm{mc}}{ }^{* *}$ with known thickness of Sisubstrate $\left(\mathrm{L}_{\mathrm{S}}=0.35 \mathrm{~mm}\right)$ and thermal diffusivity for $\mathrm{Si}$ gives $\mathrm{f}_{\mathrm{mc}}{ }^{* *} \approx 230 \mathrm{~Hz}$.

The increase of $\varphi_{\pi 1}$ value with increasing $\mathrm{f}_{\mathrm{m}}$ and approaching $\varphi_{\pi 1}$ to $\varphi_{\pi 2}$ corresponds to increase of $\omega_{\mathrm{m}} R_{\mathrm{e}} \mathrm{C}_{\mathrm{e}}$ value and the transition from $\mathrm{U}_{\pi 1}$ mode to $\mathrm{U}_{\pi 2}$ mode
The increase of $\varphi_{\pi 1}$ value with increasing $\mathrm{f}_{\mathrm{m}}$ and approaching $\varphi_{\pi 1}$ to $\varphi_{\pi 2}$ corresponds to increase of $\omega_{\mathrm{m}} R_{\mathrm{e}} \mathrm{C}_{\mathrm{e}}$ value and the transition from $U_{\pi 1}$ mode to $U_{\pi 2}$ mode when $\mathrm{U}_{\pi 1}\left(\mathrm{f}_{\mathrm{m}}\right)$ and $\mathrm{U}_{\pi 2}\left(\mathrm{f}_{\mathrm{m}}\right)$ dependences coincide with each other (see Figs $2 \mathrm{a}$ and $2 \mathrm{~b}$ ). The evaluation of the frequency of this transition $\mathrm{f}_{\mathrm{mt}}$ estimated by the known $\mathrm{R}_{\mathrm{L}}, \mathrm{R}_{\mathrm{s}}$ and $\mathrm{C}_{\mathrm{s}}$ values gives $\mathrm{f}_{\mathrm{mt}} \approx 150 \mathrm{~Hz}$.

The critical frequencies $\mathrm{f}_{\mathrm{mc}}{ }^{*}, \mathrm{f}_{\mathrm{mc}}{ }^{* *}$ and $\mathrm{f}_{\mathrm{mt}}$ are within the limits of considered peculiarities of $\mathrm{U}_{\pi 1,2}\left(\mathrm{f}_{\mathrm{m}}\right)$ and $\varphi_{\pi 1,2}\left(f_{m}\right)$.

At higher $\mathrm{f}_{\mathrm{m}}$ values the pyroelectric voltage mode for the film on the substrate is realized and the dependence $\mathrm{U} \pi_{2}\left(\mathrm{f}_{\mathrm{m}}\right)$ approaches to that given by formulae (4b). The deviation of $U \pi\left(f_{m}\right)$ behaviour from one corresponding to (4) may be connected with the frequency dependence of hopping electrical conduction $\mathrm{G}_{\mathrm{s}} \propto \mathrm{f}^{\mathrm{n}}$ (were $\mathrm{n} \sim 1$ ), characteristic for disordered systems [7].

\subsection{Double Element Position Sensitive PDR}

Obtained coordinate dependences $\mathrm{U}_{\pi}(x)$ and $\varphi_{\pi}(x)$ for different configurations of the electrodes of SE position sensitive PDR are presented in Fig. 3. For small $x$ values the linear region of $\mathrm{U} \pi(x)$ is observed in accordance with (5) - (7). With increasing $x$ the deviation from 


\section{S.L. Bravina et al.: Thin film PZT-Si structure with quadrant-diagonal electrode system...}

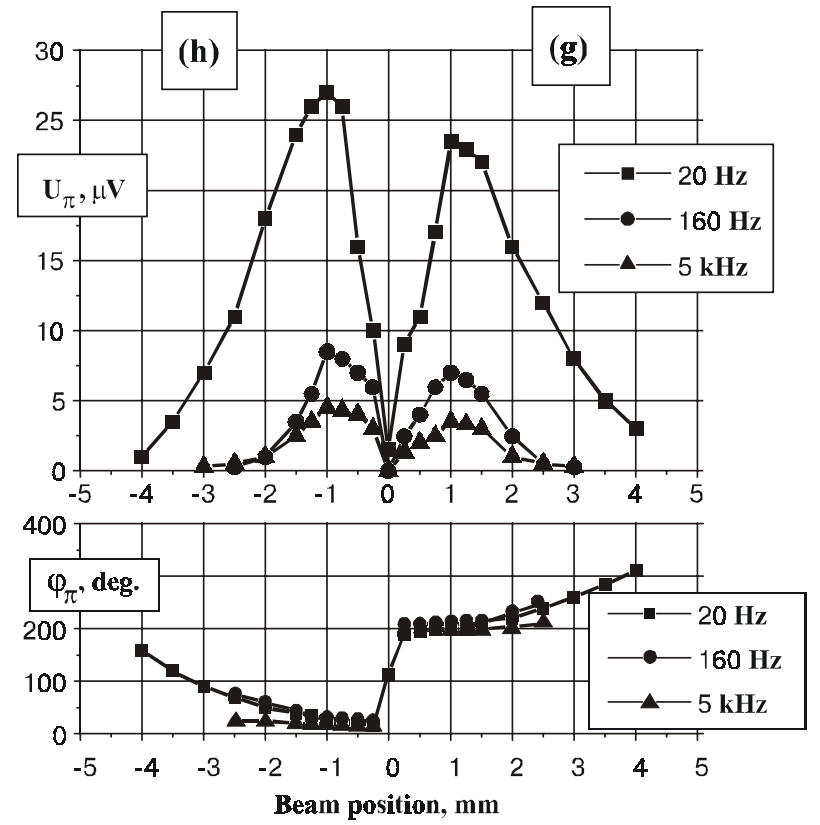

a)
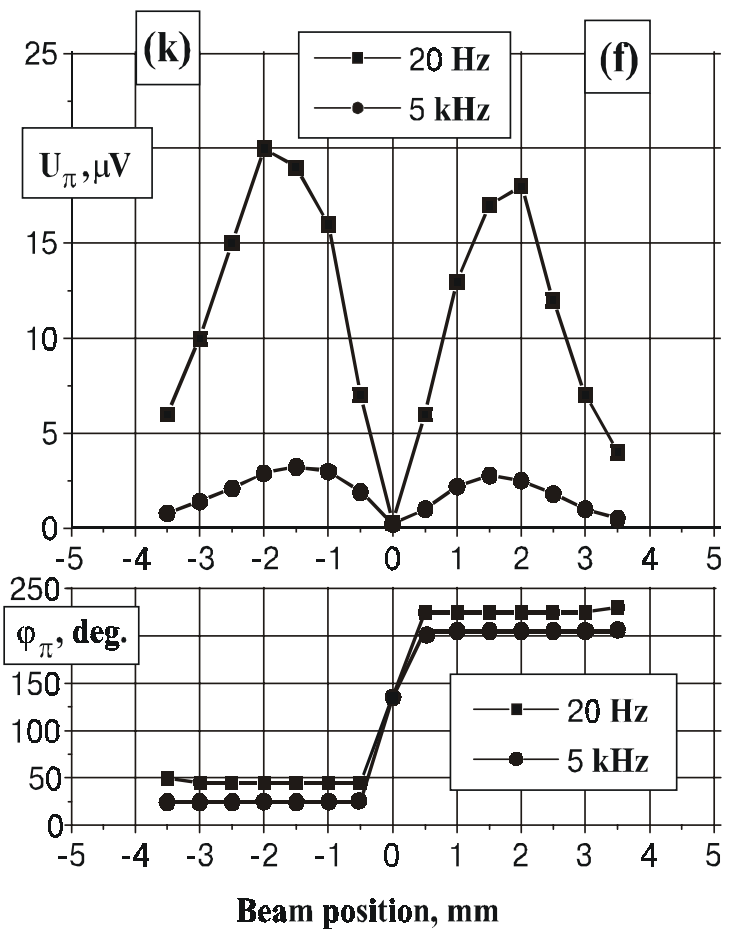

c)

Fig. 3. The dependences of $U_{\pi 2}\left(x_{0}\right)$ and $\varphi_{\pi 2}\left(x_{0}\right)$ for different configurations of the electrodes of SE position-sensitive PDR:

a - side-to-side configuration for g- and h- electrodes;

$\mathrm{b}$ - diagonal configuration for $\mathrm{f}$ - and g- electrodes;

$\mathrm{c}$ - top-to-top triangle configuration for $\mathrm{f}$ - and $\mathrm{k}$ - electrodes.
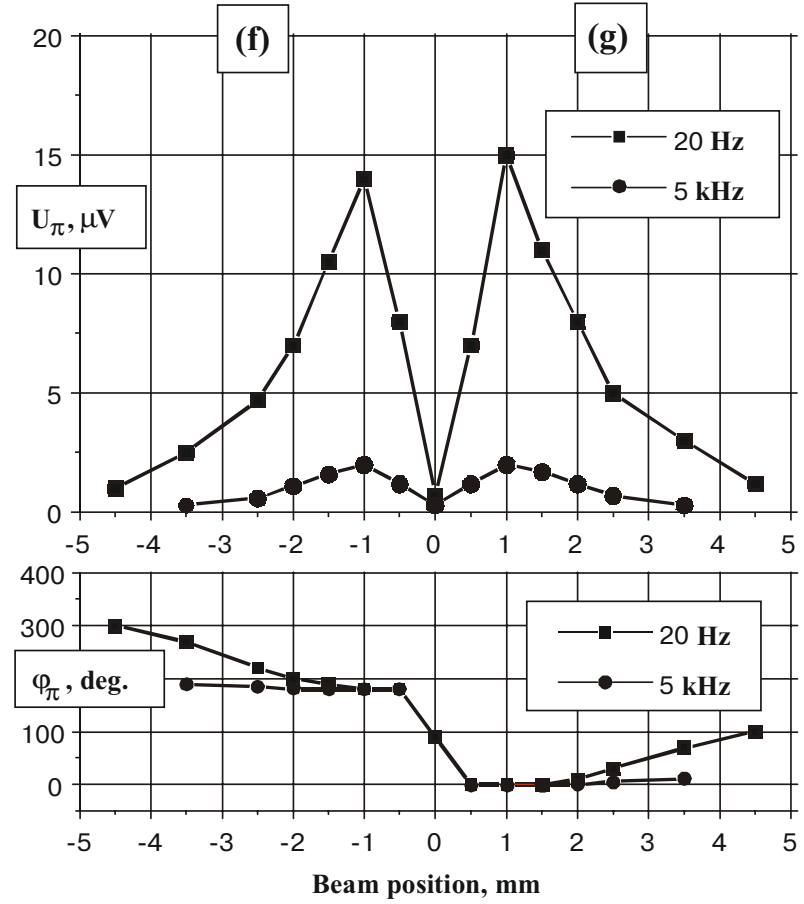

b)

linearity corresponds to decrease of position responsivity. The decrease of $\mathrm{U}_{\pi}(x)$ under large $x$ values is the consequence of falling outside the limits of the electrode region by the probe-beam. The increase of $\varphi_{\pi}$ observed at that corresponds to the increase of time and delay of the heat flux under its passing along the surface of PZT film from the energetic centre of the probe beam to the SE electrode.

Asymmetry of the position characteristics is obviously connected with the difference of R, C and $\gamma$ of each element of the investigated pair in consequence of the different value of the unipolarity degree.

As it follows from the obtained expressions for position characteristics the dynamic interval (the length of the linear region) is maximal for systems of diagonal and triangular configurations. But systems of side-to-side configuration possess maximal slope of transformation (maximal value of position responsivity). So, obtained experimental characteristics in Fig. 3 agree with those expected from (5) - (7) for the corresponding systems.

\section{Summary}

The main characteristics of position-sensitive systems of PDR with SE based on PZT film on Si-substrate were investigated for the first time by photopyromodulation method.

The results of pyroelectric undersurface probing and "along surface" pyroelectric scanning show that the investigated quadrant-diagonal system of SE makes it possible to examine all main types of differential position sensitive PDR. 


\section{S.L. Bravina et al.: Thin film PZT-Si structure with quadrant-diagonal electrode system...}

By means of paired combinations of SE were realized different 2-element single-coordinate PDR variants.

The experimental results are in an agreement with those expected from the calculations performed for the corresponding systems.

\section{References}

1. T. A. Perls and B. J. Matters, Thermal power measurement by means of pyroelectric ceramic transducers // Bull. Amer. Phys. Soc., Ser. II (4), 6, pp. 362 -363 (1959).

2. A. Van der Ziel, Pyroelectric response and $D^{*}$ of thin pyroelectric films on a substrate, //J. Appl. Phys, 44(2), pp. 546-549 (1973).
3. L. S. Kremenchugsky and O. V. Roitsina, Pyroelectric Detectors of Radiation, (in Russian), Naukova Dumka, Kiev, (1979)

4. J. C. Burfoot and G. W. Taylor, Polar Dielectrics and Their Applications, Macmillan Press, LTD London-New Jersey, (1979).

5. S. L. Bravina, N. V. Morozovsky and A. A. Strokach, Pyroelectricity: Some Physical and Application Aspects, // Proceedings of SPIE, 3182, pp.85-99 (1997),.

6. S. L. Bravina, L. S. Kremenchugsky, N. V. Morozovsky et.al., Investigation of Phase Transitions in $\mathrm{Ag}_{3} \mathrm{AsS}_{3}$ and $\mathrm{Ag}_{3} \mathrm{SbS}_{3}$ by Method of Dynamic Pyroelectric Effect, Preprint No. 26, Inst. of Phys. of Acad. Sci. of Ukraine, Kiev (1982).

7. H. Bottger and W. Bryksin, Hopping Conduction in Solids, Academic Verlag, Berlin, (1985). 